\title{
Self-reported natural history of recurrent prolapse among women presenting to a tertiary care center
}

\author{
Payton Johnson a , Kindra A. Larson ${ }^{\text {a,b }}$, Yvonne Hsu ${ }^{\text {a,b }}$, Dee E. Fenner ${ }^{\text {a,b }}$, \\ Daniel Morgan ${ }^{\mathrm{a}, \mathrm{b}}$, John O.L. DeLancey ${ }^{\mathrm{a}, \mathrm{b}, *}$ \\ a Pelvic Floor Research Group, University of Michigan, Ann Arbor, USA \\ b Division of Gynecology, Department of Obstetrics and Gynecology, University of Michigan, Ann Arbor, USA
}

\section{A R T I C L E I N F O}

\section{Article history:}

Received 29 February 2012

Received in revised form 6 July 2012

Accepted 25 September 2012

\section{Keywords:}

Prolapse

Recurrent pelvic organ prolapse

Surgical failures

\begin{abstract}
A B S T R A C T
Objective: To describe the characteristics of recurrent pelvic organ prolapse (POP). Methods: A convenience sample of patients presenting with recurrent POP symptoms between October 2007 and February 2010 completed questionnaires. The survey focused on timing of recurrence(s), symptoms, and demographics. Results: Ninety-seven women completed questionnaires. Thirty-four (35.1\%) had undergone multiple prior treatments. Overall, 23 of 76 (30.3\%) women had not informed their surgeon of the recurrence. Twentyseven of $59(45.8 \%)$ women reported that their symptoms were the same as before treatment, whereas 23 of $59(39.0 \%)$ reported more severe symptoms. POP was considered to be persistent if symptoms returned within 3 months, and recurrent if symptom relief exceeded 3 months. After primary surgery, 28 of 79 (35.4\%) cases were considered to be persistent, whereas 51 (64.6\%) cases were recurrent. Similar percentages were seen after second and third treatments. Conclusion: Overall, 35\% of participants experienced early return of symptoms. Almost one-third of participants had not informed their surgeon of the recurrence, indicating that there may not be an accurate self-assessment of outcome in the absence of careful follow-up.

(c) 2012 International Federation of Gynecology and Obstetrics. Published by Elsevier Ireland Ltd. All rights reserved.
\end{abstract}

\section{Introduction}

Symptomatic pelvic organ prolapse (POP), which is defined as the downward descent of female pelvic organs, is estimated to affect $2.9 \%$ of women in the USA [1]. One in 10 women in the USA chooses surgical management for either urinary incontinence or POP [2], which in some cases is persistent or recurrent. Retrospective and prospective observational studies have cited overall recurrence rates ranging from $13 \%$ to $58 \%$ [3-5]. Given these high rates, there is surprisingly limited information in the literature regarding the natural history and timing of recurrent POP.

The aim of the present study was to determine the following among women with recurrent prolapse confirmed on physical examination: how many had not reported the return of symptoms to their original surgeon; whether the symptoms they attributed to recurrent prolapse were similar in severity to the symptoms that were present preoperatively; and the length of time prior to them becoming aware of prolapse recurrence.

\footnotetext{
* Corresponding author at: 1500 East Medical Center Dr., Women's Hospital L4100, Ann Arbor, MI 48109-5276, USA. Tel.: +1 734647 5866; fax: +1 7346479272. E-mail address: delancey@med.umich.edu (J.O.L. DeLancey).
}

\section{Materials and methods}

The present convenience sample study was conducted at the urogynecology clinic of the University of Michigan, Ann Arbor, USA, which is a tertiary care center. Patients presenting to the urogynecology clinic for recurrent POP were recruited after identification in 1 of 2 ways. First, women presenting with confirmed recurrent prolapse (defined as any compartment at or below the hymen on physical examination) between February 1, 2008, and February 28, 2010, were identified at the time of initial clinical evaluation, and if research personnel were available a questionnaire was administered (Supplementary Material S1). Second, because convenience sampling was used and research personnel were not always available, surgical records were then retrospectively reviewed for women who underwent surgery for recurrent prolapse at the study institution between October 1,2007 , and February 28, 2010, who had not already completed the questionnaire (Supplementary Material S2). Inclusion criteria required that women had at least 1 prior episode of POP managed with operative treatment and confirmed recurrence of POP on physical examination. The recurrent POP at the time of presentation was regarded as the index prolapse. The study was approved by the Institutional Review Board for Human Subject Research (HUM00017850) of the University of Michigan. The completion and return of the questionnaire from patients implied consent. 
Patients were asked to complete a questionnaire and characterize the index POP, as well as reflect on past recurrences. The survey was generated by several of the authors-using questions that were typically captured on initial evaluation-and focused on information related to timing of recurrence(s), symptoms of index POP recurrence, and demographic characteristics (body mass index, parity, hysterectomy, and menopausal status).

The initial questionnaire was screened among a pilot group of women, then updated to enhance clarity based on their feedback. Further characterization of POP and the timeline of recurrence was achieved via review of physician notes and electronic medical records by research assistants. When available, POP Quantification System (POP-Q) values were used to define prolapse within the anterior (Ba), posterior (Bp), and apical (C) compartments. Prolapse was defined as a POP-Q value of greater than or equal to 0 . The timeline of recurrence was established from the patient survey and was based on the answer to the following question: "Did you have any period of time after your most recent failed surgery for prolapse during which you were not bothered by the bulge? If yes, for how long?" Recurrent prolapse was defined subjectively by the patient for all recurrences and was objectively confirmed for the index recurrence as descent of any vaginal compartment below the hymen on clinical examination.

Data were evaluated with SAS version 9.1 (SAS Institute, Cary, NC, USA) using descriptive statistics, unpaired and paired $t$ tests, and McNemar test. $P<0.05$ was considered to be statistically significant.

\section{Results}

In total, 106 questionnaires were completed and collected. Nine were excluded because patient identity could not be confirmed in order to perform chart substantiation of the data. Thus, 97 completed questionnaires were available for analysis. Not all women answered each question, however, so data are presented with the denominator representing the number who answered a particular question. The participating women were all parous, predominantly white, and postmenopausal, with prior hysterectomy (two-thirds were for prolapse) and confirmed prolapse on examination. Before presentation, all patients had undergone at least 1 surgical treatment for prolapse, and 34 (35.1\%) had undergone multiple prior treatments (Table 1 ).

In total, 80 of $94(85.1 \%)$ participants reported that they selfdiscovered the index prolapse, whereas the remaining 14 (14.9\%) were diagnosed by a physician. Overall, 23 of 76 (30.3\%) women reported that they had not informed their previous surgeon of the recurrence. At presentation to the study center, 90 (92.8\%) patients had a documented POP-Q score confirming at least 1 compartment prolapse, as defined above. Of the participants with documented POP-Q values, 55 of $89(61.8 \%)$ had an anterior prolapse, 37 of $90(41.1 \%)$ had a posterior prolapse, and 30 of 90 (33.3\%) had an apical prolapse (Fig. 1). As determined by POP-Q values, 46 (51.1\%) had 1, 27 (30.0\%) had 2 , and $17(18.9 \%)$ had 3 prolapsed compartments. The POP-Q values between the patient- and the doctor-discovered populations were not statistically different when comparing specific compartments (Ba $[P=0.205]$; $\mathrm{Bp}[P=0.231]$; and $\mathrm{C}[P=0.220]$ ) or maximal descent $(P=0.970)$.

In addition to the symptoms due to the prolapse itself, other pelvic floor symptoms at the index POP were described by 91 participants. The most common non-prolapse symptoms associated with prolapse return were incomplete emptying of bowel $(n=51$ [56.0\%]), urinary incontinence $(n=49[53.8 \%])$, low back pain $(n=45[49.5 \%])$, constipation ( $n=38$ [41.8\%]), and dyspareunia ( $n=36$ [39.6\%]) (Fig. 2). Overall, 47 of 89 (52.8\%) participants reported that their index symptoms were consistent with symptoms they experienced with their previous prolapse. Fifty-nine women reported on the severity of their symptoms; 27 (45.8\%) stated that they were the same as before treatment and 23 (39.0\%) stated that they were more severe.
Table 1

Participant demographics $(n=97){ }^{\text {a }}$

\begin{tabular}{ll}
\hline Characteristic & Value \\
\hline Age at presentation, $\mathrm{y}$ & $62.6 \pm 9.8$ \\
Age at initial occurrence, y & $50.7 \pm 14.4$ \\
BMI & $29.7 \pm 18.0$ \\
Parity & $2.9 \pm 1.3$ \\
Hysterectomy & \\
No & $4(4.1)$ \\
Yes & $93(95.9)$ \\
$\quad$ Prolapse & $62(67.0)$ \\
$\quad$ Other & $31(33.0)$ \\
Menopausal $(\mathrm{n}=96)$ & \\
No & $8(8.3)$ \\
Yes & $88(91.7)$ \\
Race & \\
White & $93(95.9)$ \\
African American & $3(3.1)$ \\
Asian & $1(1.0)$ \\
Prolapse & $97(100.0)$ \\
Total number of previous surgeries & \\
1 & $63(64.9)$ \\
2 & $25(25.8)$ \\
3 & $6(6.2)$ \\
$\geq 4$ & $3(3.1)$ \\
\hline
\end{tabular}

Abbreviation: BMI, body mass index (calculated as weight in kilograms divided by the square of height in meters).

${ }^{\text {a }}$ Values are given as mean \pm SD or number (percentage).

b Confirmed by physician at physical examination.

Participants were stratified as having persistent prolapse if return of symptoms occurred within 3 months of previous surgery, and recurrent prolapse if relief of symptoms was present for at least 3 months after surgery. Fig. 3 shows the percentage of participants in each category after each surgical treatment. Patient recall of

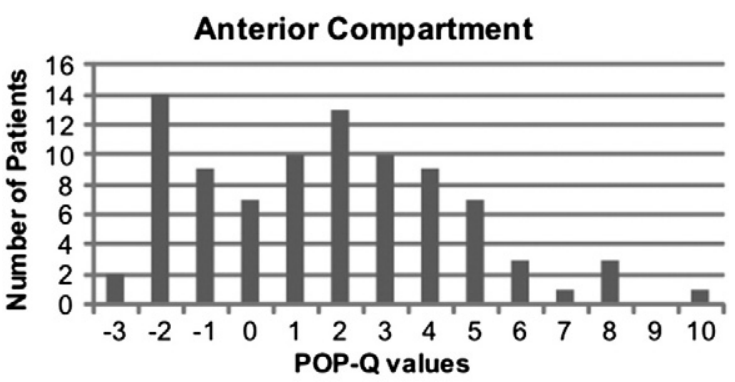

Posterior Compartment

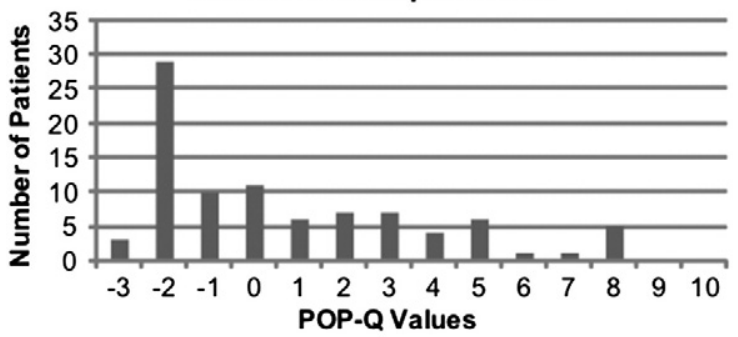

Apical Compartment

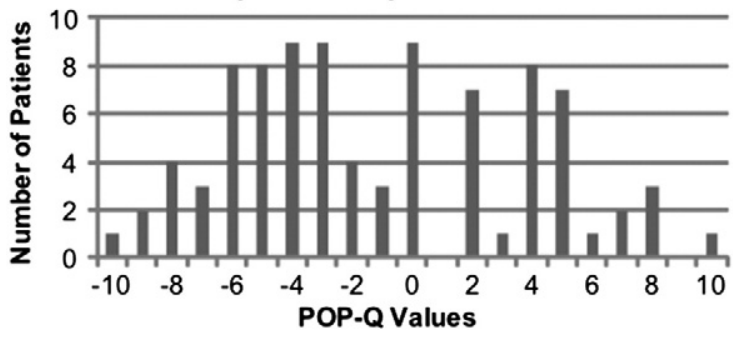

Fig. 1. Histograms of compartment Pelvic Organ Prolapse Quantification System values. 


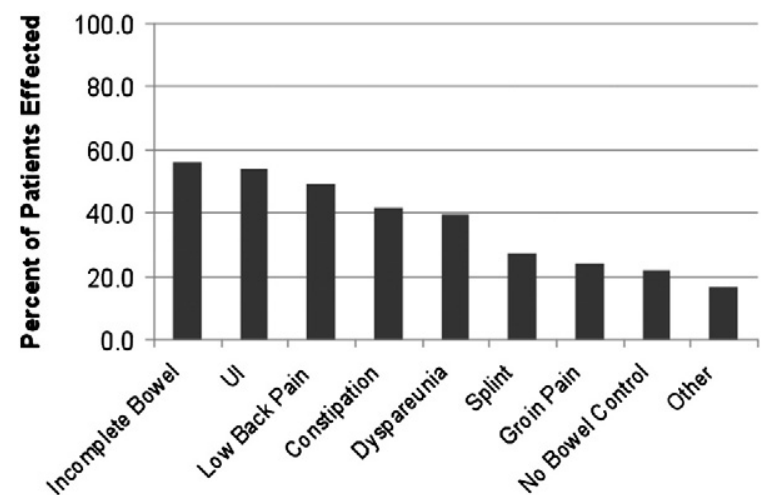

Characteristic Symptom of Index Recurrent POP

Fig. 2. Most commonly experienced symptoms of patients at presentation.

symptom return showed that, after primary surgery for POP, 28 of 79 (35.4\%) women were categorized as having persistent prolapse and 51 (64.6\%) had recurrent POP, with a mean 69.1 months of relief. Similar percentages were seen for persistence versus recurrence after second and third treatments. When evaluating first, second, and third treatments, there was no significant difference in the proportions of women who experienced return of symptoms within 3 months $(P=0.980$; McNemar test $)$.

Table 2 shows mean time to return of symptoms and next surgical treatment for the entire cohort (i.e. recurrent and persistent groups). Although there seemed to be a trend toward shorter time intervals with each successive treatment, paired $t$ tests did not show significant differences between the groups for return of symptoms $(P=0.16$ for primary and secondary; $P=0.13$ for secondary and tertiary) or subsequent treatment $(P=0.25$ for primary and secondary; $P=0.24$ for secondary and tertiary). Fig. 4 shows return of symptoms as a function of time, emphasizing the large proportion of patients with early return of symptoms.

\section{Discussion}

There are 3 key findings from the present study of women seeking care for recurrent prolapse: regardless of the number of prior treatment failures, symptoms returned within 3 months of the operation in 35\% of cases; almost one-third of patients reported that they had not informed their previous surgeon of symptom recurrence; and the majority of women felt that their symptoms were either the same as or worse than before treatment. Although mean time to return of symptoms and time to next treatment seemed to decrease

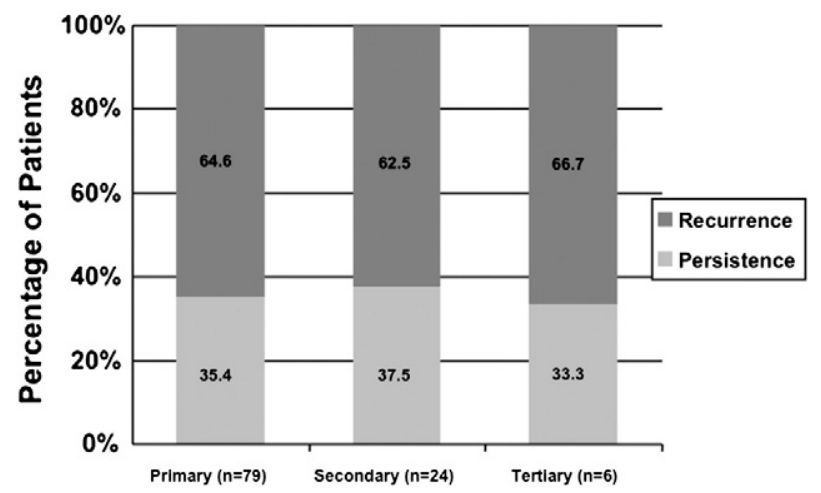

Fig. 3. Percentages of patients with return of symptoms within 3 months of treatment (persistence group) and those who experienced more than 3 months of relief (recurrence group)
Table 2

Patient recall of symptom return and treatment timeline.

\begin{tabular}{lll}
\hline Surgery & Return of symptoms, mo & Interval to next surgery, mo \\
\hline Primary & & \\
Mean \pm SD & $45.1 \pm 86.9^{\mathrm{a}}$ & $89.3 \pm 106.3^{\mathrm{d}}$ \\
$\quad$ Median (range) & $6(0-480)^{\mathrm{a}}$ & $48(2-555)^{\mathrm{d}}$ \\
Secondary & & \\
$\quad$ Mean \pm SD & $24.5 \pm 36.8^{\mathrm{b}}$ & $71.7 \pm 77.9^{\mathrm{e}}$ \\
$\quad$ Median (range) & $6(0-120)^{\mathrm{b}}$ & $35(4-341)^{\mathrm{e}}$ \\
Tertiary & & \\
Mean \pm SD & $14.8 \pm 13.6^{\mathrm{c}}$ & $29.9 \pm 25.2^{\mathrm{f}}$ \\
Median (range) & $15(0-36)^{\mathrm{c}}$ & $23.5(4-75)^{\mathrm{f}}$ \\
\hline
\end{tabular}

a $\mathrm{n}=79$.

${ }^{\mathrm{b}} \mathrm{n}=24$

c $\mathrm{n}=6$.

${ }^{\mathrm{d}} \mathrm{n}=97$

$\mathrm{e} n=31$.

f $\mathrm{n}=8$.

with each successive operation, there was too much variability within the sample size for these results to be statistically significant.

The timing of recurrent prolapse has been detailed in several studies based on clinical examination at follow-up appointments, intervals between treatments, and reoperation rates calculated from records [5-7]. Price et al. [6] reported that more than half of patients undergoing their first reoperation for recurrent prolapse did so in the first 3 years, and Fialkow et al. [7] reported a mean time to reoperation of 4.15 years [7]. The present median of 4 years to reoperation after primary surgical treatment is consistent with these published data.

Timing of early recurrence is sparsely reported in the literature. Based on reoperation rates, Clark et al. [4] plotted survival curves of 2 populations (first operation vs previous operative treatments) but did not detail early recurrences. Price et al. [6] reported that the majority of their population experienced recurrence within 3 years [6]; however, the present study addressed the return of symptoms within the first 3 months after surgery.

In the present study, there were time differences between a patient's perception of prolapse return and when treatment was sought for recurrent prolapse, indicating that the date a patient returns for retreatment or the date of reoperation may not be good surrogates for documenting when prolapse actually returned. The study aimed to distinguish between persistence and recurrence of POP, using return of symptoms as the marker. The early return of symptoms indicates persistence of prolapse or failure of a previous operative approach to achieve even short-term success, thus questioning the current ability to address properly the initial problem causing POP.

At the study center, almost one-third of participants had not reported the return of symptoms to their primary surgeon, indicating that surgeons may not know that such failures have occurred. In addition to migration issues, the literature highlights many barriers to seeking further treatment. Attitudes toward aging, incontinence, treatment, and health professionals are all reasons for some women not seeking further treatment [8]-often leaving the primary surgeon unaware of any problems. Limited postoperative information for the primary surgeon leads to less self-awareness of surgical failure rates and is rarely discussed in the literature.

The most common symptoms reported by the study population were incomplete emptying of bowel, urinary incontinence, low back pain, constipation, and dyspareunia. Importantly, most participants reported symptoms of similar or worse severity compared with prior episodes of prolapse. Because there were no objective data regarding prior prolapse characteristics of patients, the reported data could not be correlated with prolapse compartment or prolapse severity. This is unfortunate because prolapse and its associated symptoms have been vigorously investigated in the literature, resulting in contrasting evidence. Urinary and defecatory obstructive symptoms 


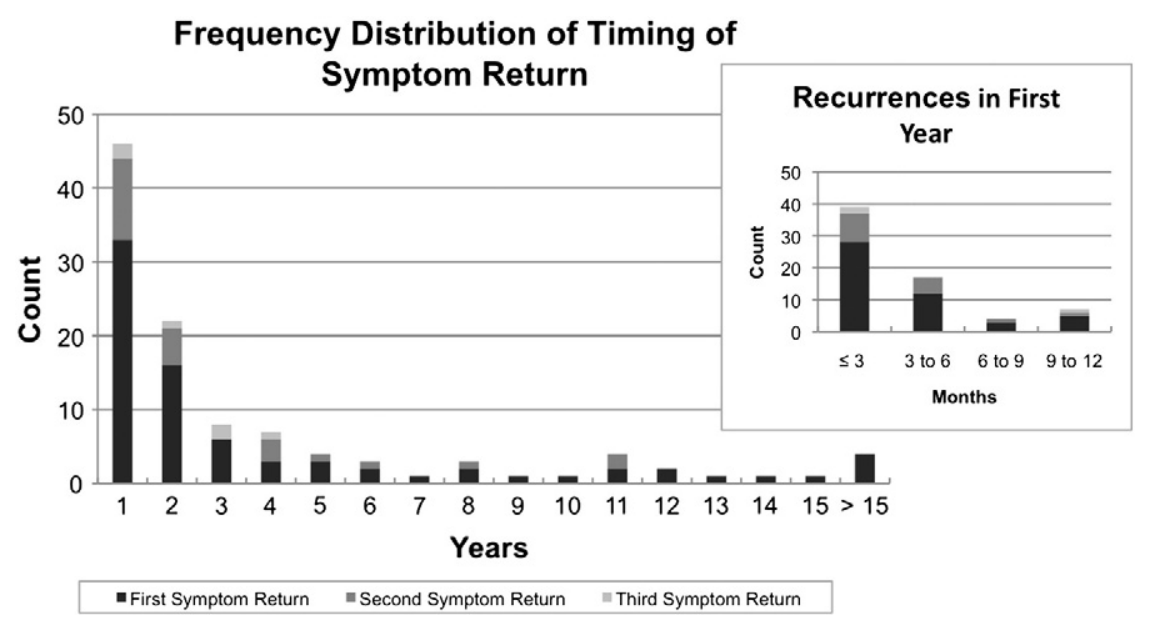

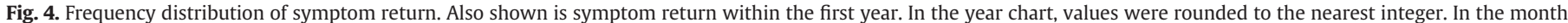
chart, they were placed in the interval they fell into (no rounding occurred).

have been shown to be associated with POP $[9,10]$; some studies have even cited specific symptom correlations with prolapse severity $[11,12]$. In the study by Erekson et al. [12], the most commonly experienced symptoms were dyspareunia, incomplete voiding, constipation, urinary frequency, and urgency. Other groups have shown that common symptoms of low back and groin pain are not associated with prolapse $[13,14]$ and that frequency and voiding difficulties, bowel movements, and the need for splinting do not correlate with specific compartments of prolapse [15].

There is a multitude of research on the risk factors associated with POP and recurrent POP. It has been reported that previous reconstructive surgery is a risk factor for recurrence $[4,16]$; this information correlates well with the trend in the present study that time to return of symptoms and mean time between treatments decrease with each successive operation (although the present data were not statistically significant).

Although recall bias must be considered a constraint of the present study, the results are still valuable because many clinical decisions are based on patients' reported histories in conjunction with objective physical findings. The retrospective nature of the study limited the opportunity to conduct survivability tests. In addition, objective POP-Q data were not available for all previous failures, meaning that a compartment failure analysis could not be performed; this information would be helpful and deserves further study. Finally, the present study involved a convenience sample of a predominately white population (disqualifying any interracial comparisons) at a tertiary-care referral center, so the generalizability of the results is limited. However, although the findings may not be applicable to the general population, they are still important in terms of the assessment of referral clinic cohorts.

Despite the limitations, the present study indicates that-in the absence of careful follow-up-surgeons may have inaccurate selfassessments of success. It also highlights the high percentage of early recurrence, thus indicating that current surgical techniques do not properly address the causes of POP and providing momentum to the quest for better understanding of the mechanisms of prolapse.

Supplementary data to this article can be found online at http:// dx.doi.org/10.1016/j.ijgo.2012.07.024.

\section{Acknowledgments}

Support was provided by the National Institute of Child Health and Human Development (grant R01 HD 38665), with additional investigator financial support from the Office for Research on Women's
Health SCOR on Sex and Gender Factors Affecting Women's Health (1 P50 HD044406).

\section{Conflict of interest}

The University of Michigan receives research grant funds from American Medical Systems, Kimberly Clark, and Johnson \& Johnson in partial support of Dr. DeLancey's salary. D.E.F. participates in clinical trials with American Medical Systems.

\section{References}

[1] Nygaard I, Barber MD, Burgio KL, Kenton K, Meikle S, Schaffer J, et al. Prevalence of symptomatic pelvic floor disorders in US women. JAMA 2008;300(11):1311-6.

[2] Boyles SH, Weber AM, Meyn L. Procedures for pelvic organ prolapse in the United States, 1979-1997. Am J Obstet Gynecol 2003;188(1):108-15.

[3] Olsen AL, Smith VJ, Bergstrom JO, Colling JC, Clark AL. Epidemiology of surgically managed pelvic organ prolapse and urinary incontinence. Obstet Gyneco 1997;89(4):501-6.

[4] Clark AL, Gregory T, Smith VJ, Edwards R. Epidemiologic evaluation of reoperation for surgically treated pelvic organ prolapse and urinary incontinence. Am J Obstet Gynecol 2003;189(5):1261-7.

[5] Whiteside JL, Weber AM, Meyn LA, Walters MD. Risk factors for prolapse recurrence after vaginal repair. Am J Obstet Gynecol 2004;191(5):1533-8.

[6] Price N, Slack A, Jwarah E, Jackson S. The incidence of reoperation for surgically treated pelvic organ prolapse: an 11-year experience. Menopause Int 2008:14(4):145-8.

[7] Fialkow MF, Newton KM, Weiss NS. Incidence of recurrent pelvic organ prolapse 10 years following primary surgical management: a retrospective cohort study. Int Urogynecol J Pelvic Floor Dysfunct 2008;19(11):1483-7.

[8] Basu M, Duckett JR. Barriers to seeking treatment for women with persistent or recurrent symptoms in urogynecology. BJOG 2009;116(5):726-30.

[9] Bradley CS, Kennedy CM, Nygaard IE. Pelvic floor symptoms and lifestyle factors in older women. J Womens Health (Larchmt) 2005;14(2):128-36.

[10] Weber AM, Walters MD, Ballard LA, Booher DL, Piedmonte MR. Posterior vaginal prolapse and bowel function. Am J Obstet Gynecol 1998;179(6 Pt 1):1446-9.

[11] Ellerkmann RM, Cundiff GW, Melick CF, Nihira MA, Leffler K, Bent AE. Correlation of symptoms with location and severity of pelvic organ prolapse. Am J Obstet Gynecol 2001;185(6):1332-7.

[12] Erekson EA, Kassis NC, Washington BB, Myers DL. The Association Between Stage II or Greater Posterior Prolapse and Bothersome Obstructive Bowel Symptoms. Female Pelvic Med Reconstr Surg 2010;16(1):59-64

[13] Swift SE, Tate SB, Nicholas J. Correlation of symptoms with degree of pelvic organ support in a general population of women: what is pelvic organ prolapse? Am J Obstet Gynecol 2003;189(2):372-7.

[14] Heit M, Culligan P, Rosenquist C, Shott S. Is pelvic organ prolapse a cause of pelvic or low back pain? Obstet Gynecol 2002;99(1):23-8.

[15] Ghetti C, Gregory WT, Edwards SR, Otto LN, Clark AL. Pelvic organ descent and symptoms of pelvic floor disorders. Am J Obstet Gynecol 2005;193(1):53-7.

[16] Vakili B, Zheng YT, Loesch H, Echols KT, Franco N, Chesson RR. Levator contraction strength and genital hiatus as risk factors for recurrent pelvic organ prolapse. Am J Obstet Gynecol 2005;192(5):1592-8. 